\title{
SDC13 infrared dark clouds: Longitudinally collapsing filaments?
}

\author{
N. Peretto ${ }^{1,2}$, G. A. Fuller ${ }^{3}$, Ph. André ${ }^{2}$, D. Arzoumanian ${ }^{4}$, V. M. Rivilla ${ }^{5}$, S. Bardeau ${ }^{6}$, S. Duarte Puertas $^{7}$, \\ J. P. Guzman Fernandez ${ }^{7}$, C. Lenfestey ${ }^{3}$, G.-X. Li ${ }^{8}$, F. A. Olguin, ${ }^{9,10}$, B. R. Röck ${ }^{11,12}$, H. de Villiers ${ }^{13}$, and J. Williams ${ }^{3}$ \\ 1 School of Physics \& Astronomy, Cardiff University, Queens Buildings, The parade, Cardiff CF24 3AA, UK \\ e-mail: Nicolas.Peretto@astro.cf.ac.uk \\ ${ }^{2}$ Laboratoire AIM, CEA/DSM-CNRS-Universté Paris Diderot, IRFU/Service d'Astrophysique, C. E. Saclay, \\ 91191 Gif-sur-Yvette Cedex, France \\ 3 Jodrell Bank Centre for Astrophysics, School of Physics and Astronomy, University of Manchester, Manchester, M13 9PL, UK \\ 4 IAS, CNRS (UMR 8617), Université Paris-Sud, Bâtiment 121, 91400 Orsay, France \\ 5 Centro de Astrobiología (CSIC-INTA), Ctra. de Torrejón-Ajalvir, km. 4, 28850 Torrejón de Ardoz, Madrid, Spain \\ ${ }^{6}$ Institut de Radioastronomie Millimétrique, 300 Rue de la piscine, 38406 Saint-Martin-d'Hères, France \\ 7 Universidad de Granada, 18071 Granada, Spain \\ 8 Max-Planck Institut für Radioastronomie, Auf dem Hügel, 69, 53121 Bonn, Germany \\ 9 School of Physics and Astronomy, University of Leeds, Leeds LS2 9JT, UK \\ ${ }_{10}$ Departamento de Astronomía, Universidad de Chile, 36-D Casilla, Santiago, Chile \\ 11 Instituto de Astrofísica de Canarias, 38200 La Laguna, Tenerife, Spain \\ 12 Universidad de la Laguna, Dept. Astrofisica, 38206 La Laguna, Tenerife, Spain \\ 13 Centre for Astrophysics Research, University of Hertfordshire, College Lane, Hatfield, Herts, AL10 9AB, UK
}

Received 28 June 2013 / Accepted 31 October 2013

\begin{abstract}
Formation of stars is now believed to be tightly linked to the dynamical evolution of interstellar filaments in which they form. In this paper we analyze the density structure and kinematics of a small network of infrared dark filaments, SDC13, observed in both dust continuum and molecular line emission with the IRAM $30 \mathrm{~m}$ telescope. These observations reveal the presence of 18 compact sources amongst which the two most massive, MM1 and MM2, are located at the intersection point of the parsec-long filaments. The dense gas velocity and velocity dispersion observed along these filaments show smooth, strongly correlated, gradients. We discuss the origin of the SDC13 velocity field in the context of filament longitudinal collapse. We show that the collapse timescale of the SDC13 filaments (from 1 Myr to 4 Myr depending on the model parameters) is consistent with the presence of Class I sources in them, and argue that, on top of bringing more material to the centre of the system, collapse could generate additional kinematic support against local fragmentation, helping the formation of starless super-Jeans cores.
\end{abstract}

Key words. stars: formation - ISM: clouds - ISM: kinematics and dynamics - ISM: structure

\section{Introduction}

In recent years, interstellar filaments have received special attention. The far-infrared Herschel space observatory revealed the ubiquity of filaments in both quiescent and active star-forming clouds. The detailed analysis of large samples of filaments identified with Herschel suggest that they represent a key stage in the formation of low-mass prestellar cores (Arzoumanian et al. 2011). In the picture proposed by André et al. (2010), these cores form out of filaments that have reached the thermal critical mass-per-unit-length, $M_{\text {line,crit }}^{\text {th }}=2 c_{\mathrm{s}}^{2} / G$ (Ostriker 1964), above

\footnotetext{
$\star$ Based on observations carried out with the IRAM $30 \mathrm{~m}$ Telescope. IRAM is supported by INSU/CNRS (France), MPG (Germany), and IGN (Spain).

$\star \star$ Figure 3 and Appendices are available in electronic form at http: //www . aanda.org

$\star \star \star$ The FITS files for the $\mathrm{N}_{2} \mathrm{H}^{+}(1-0)$ Spectra of Fig. 3 are only available at the CDS via anonymous ftp to cdsarc.u-strasbg.fr (130.79.128.5) or via

http://cdsarc.u-strasbg.fr/viz-bin/qcat?J/A+A/561/A83
}

which filaments become gravitationally unstable. Filaments with $M_{\text {line }}>M_{\text {line,crit }}^{\text {th }}$ are called supercritical filaments. While this scenario might apply to the bulk of the low-mass prestellar cores, it can hardly account for the formation of superJeans cores (e.g. Sadavoy et al. 2010) whose mass is several times higher than the local thermal Jeans mass $M_{J}^{\text {th }}\left(\sim 1 M_{\odot}\right.$ for critical $10 \mathrm{~K}$ filaments). Additional support (magnetic, kinematic) and/or significant subsequent core accretion are needed to explain the formation of cores with masses $M_{\text {core }} \gg M_{J}^{\text {th }}$.

Several high-resolution studies of filamentary cloud kinematics have been performed in the past three years towards low-mass, nearby, star-forming regions (e.g. Duarte-Cabral et al. 2010; Hacar \& Tafalla 2011; Kirk et al. 2013; Arzoumanian et al. 2013). These studies demonstrate the importance of cloud kinematics in the context of filament evolution and core formation. For instance, Kirk et al. (2013) showed that gas filamentary accretion towards the central cluster of young stellar objects (YSOs) in Serpens South could sustain the star formation rate observed in this cloud, providing enough material to constantly form new generations of YSOs. But, it is not clear 


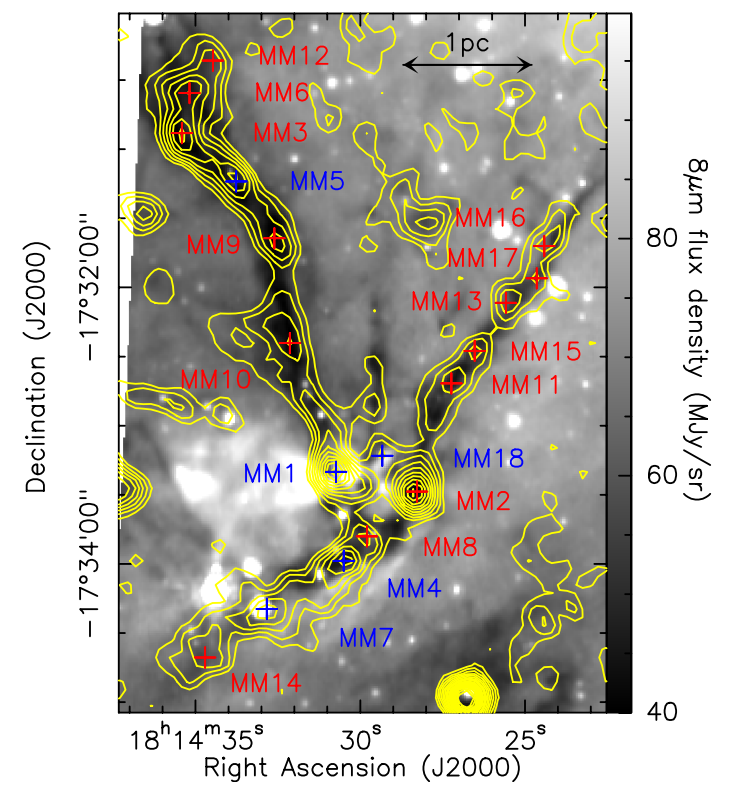

Fig. 1. Spitzer $8 \mu \mathrm{m}$ image of SDC13 in grey scale on top of which we overlaid the IRAM $30 \mathrm{~m}$ MAMBO $1.2 \mathrm{~mm}$ dust continuum contours (from $3 \mathrm{mJy} /$ beam to $88 \mathrm{mJy} /$ beam in step of $5 \mathrm{mJy} / \mathrm{beam}$ ). The positions of the identified $1.2 \mathrm{~mm}$ compact sources within the SDC13 filaments are marked as crosses, red for starless sources and blue for protostellar sources.

that these inflows have any impact at all in determining the mass of individual low-mass cores. On the high-mass side, infrared dark clouds (IRDCs) have been privileged targets (e.g. Miettinen 2012; Ragan et al. 2012; Henshaw et al. 2013; Busquet et al. 2013; Peretto et al. 2013). These sources are typically located at a distance of $\sim 4 \mathrm{kpc}$ from the Sun (Peretto \& Fuller 2010), making the analysis of filament kinematics more difficult. Thanks to the high sensitivity and resolution of ALMA, Peretto et al. (2013) showed that the global collapse of the SDC335.5790.292 IRDC is responsible for the formation of an early O-type star progenitor $\left(M \simeq 545 M_{\odot}\right.$ in $\left.0.05 \mathrm{pc}\right)$ sitting at the cloud centre.

In this paper, we present results of an IRDC (hereafter called SDC13), composed of three Spitzer dark clouds from the Peretto \& Fuller (2009) catalogue (SDC13.174-0.07, SDC13.158-0.073, SDC13.194-0.073). The near kinematical distance of SDC13 is $d=3.6( \pm 0.4) \mathrm{kpc}$ using the Reid et al. (2009) model. From $8 \mu \mathrm{m}$ extinction we estimate a total gas mass of $\sim 600 M_{\odot}$ above $N_{\mathrm{H}_{2}}>10^{22} \mathrm{~cm}^{-2}$. As for any IRDC, SDC13 shows little star formation activity, except in the central region where extended infrared emission is observed (see Fig. 1 from Peretto \& Fuller 2010). The high extinction contrast, the rather simple geometry, and the high aspect ratios of the SDC13 filaments are remarkable and provide a great opportunity to better understand the impact of filament kinematics on the formation of cores in an intermediate mass regime.

\section{Millimetre observations}

\subsection{Dust continuum data}

In December 2009 we observed SDC13 at the IRAM $30 \mathrm{~m}$ telescope using the MAMBO bolometer array at $1.2 \mathrm{~mm}$. We performed on-the-fly mapping of a $5^{\prime} \times 5^{\prime}$ region. The sky opacity at $225 \mathrm{GHz}$ was measured to be between 0.08 and $0.26 \mathrm{de}$ pending on the scan. Pointing accuracy was better than $2^{\prime \prime}$ and calibration on Uranus was better than $10 \%$. We used MOPSIC
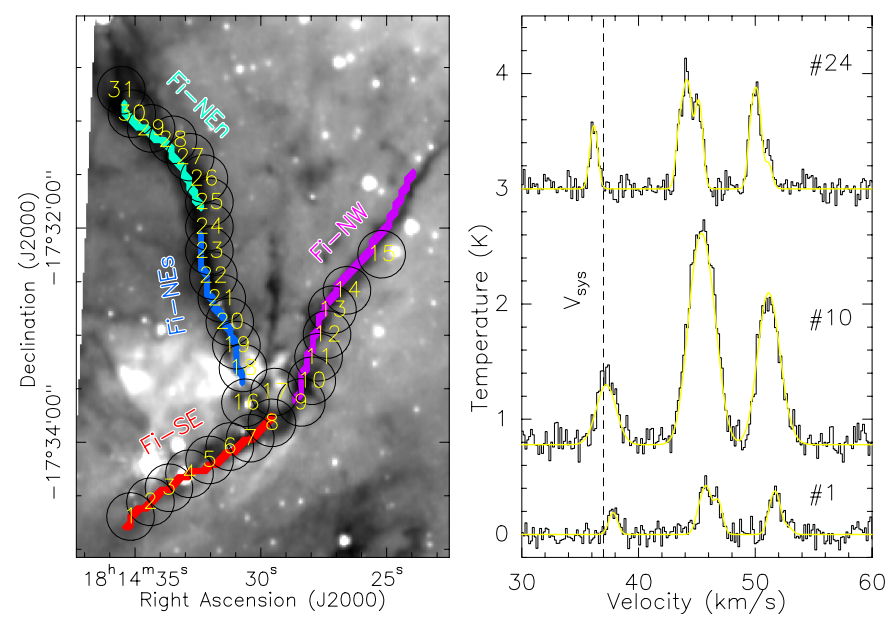

Fig. 2. Left: Spitzer $8 \mu \mathrm{m}$ image of SDC13 in grey scale on top of which we marked the 31 single-pointing positions we observed in $\mathrm{N}_{2} \mathrm{H}^{+}(1-0)$, and the skeletons of each filament (thick solid lines). The size of the black circles represents the $30 \mathrm{~m}$ beam size at $3.2 \mathrm{~mm}$. Right: examples of three $\mathrm{N}_{2} \mathrm{H}^{+}(1-0)$ spectra (displayed on $T_{a}^{*}$ temperature scale) observed at three different positions (1, 10, and 24). Their corresponding hyperfine structure fits are displayed as yellow solid lines. The remaining spectra are displayed in Fig. 3. The vertical dashed line corresponds to the systemic velocity of the cloud (i.e. $V_{\text {sys }}=37.0 \mathrm{~km} \mathrm{~s}^{-1}$ ) as measured from the isolated component of the hyperfine structure.

to reduce these MAMBO data, obtaining a final rms noise level on the reduced image of $\sim 1 \mathrm{mJy} / 11^{\prime \prime}$-beam.

We also make use of publicly available Spitzer GLIMPSE (Churchwell et al. 2009) and $24 \mu \mathrm{m}$ MIPSGAL (Carey et al. 2009) data.

\subsection{Molecular line data}

In September 2011, during the IRAM 30 m summer school, we observed SDC13 with the IRAM 30 m telescope using the EMIR heterodyne receiver. We performed 31 single-pointing observations along the SDC13 filaments in $\mathrm{N}_{2} \mathrm{H}^{+}(1-0)$, at $93.2 \mathrm{GHz}$ (i.e. $27^{\prime \prime}$ beam). The off positions, taken $5^{\prime}$ away from SDC13, were checked to ensure no emission was present there. The pointing was better than $5^{\prime \prime}$ and the sky opacity was varying between 0.3 to 2 at $225 \mathrm{GHz}$. We used the FTS spectrometer with a $50 \mathrm{KHz}$ spectral resolution. The data were reduced in CLASS $^{1}$. The resulting spectra have an rms noise varying from 0.05 to $0.1 \mathrm{~K}$ in a $0.16 \mathrm{~km} \mathrm{~s}^{-1}$ channel width.

\section{Analysis}

\subsection{Mass partition in SDC13}

The MAMBO observations of SDC13 are presented in Fig. 1. We can see that the $1.2 \mathrm{~mm}$ dust continuum emission towards SDC13 matches the mid-infrared extinction very well. In the remainder of this paper we focus only on the emission towards these dark filaments and ignore any other significant emission peaks since we do not have kinematical information for those.

We segmented the emission on this dust continuum map into four filaments: Fi-SE, Fi-NW, Fi-NEs, Fi-NEn. The skeletons of these filaments (Fig. 2-left) were obtained by fitting polynomial functions to the MAMBO emission peaks along them. The splitting of Fi-NE into two parts is due to its dynamical

http://www . iram. fr/IRAMFR/GILDAS 
Table 1. Observed properties of SDC13 filaments.

\begin{tabular}{|c|c|c|c|c|}
\hline Name & $\begin{array}{c}\text { Sizes } \\
\left({ }^{\prime \prime} \times{ }^{\prime \prime}\right)\end{array}$ & $\begin{array}{l}\text { PA } \\
\left({ }^{\circ}\right)\end{array}$ & $\begin{array}{l}R_{\text {eff }} \\
\left({ }^{\prime \prime}\right)\end{array}$ & $\begin{array}{c}F_{1.2 \mathrm{~mm}}^{\mathrm{int}} \\
(\mathrm{Jy})\end{array}$ \\
\hline Fil-NEn & $104 \times 20$ & +38 & 30.6 & $0.259 \pm 0.032$ \\
\hline Fil-NEs & $95 \times 19$ & +19 & 29.5 & $0.213 \pm 0.030$ \\
\hline Fil-NW & $152 \times 16$ & -31 & 29.6 & $0.198 \pm 0.031$ \\
\hline Fil-SE & $107 \times 22$ & -56 & 32.8 & $0.219 \pm 0.032$ \\
\hline
\end{tabular}

Notes. Column 1: filament name; Col. 2: non-deconvolved filament length and filament width, angular size uncertainties are $\pm 1^{\prime \prime}$; Col. 3 : filament position angle; Col. 4: filament geometric radius; Col. 5: $1.2 \mathrm{~mm}$ dust continuum flux.

structure (cf. Sect. 3.2). These filaments are barely resolved in our MAMBO map and are responsible for most of the $1.2 \mathrm{~mm}$ flux in the region. Although ground-based dust continuum observations filter out all emission whose spatial scale is larger than the size of the bolometer array ( $2^{\prime}$ for MAMBO), inspection of Herschel Hi-GAL observations (Molinari et al. 2010) shows that the SDC13 mass is indeed mostly concentrated within the filaments. The observed properties of the filaments (as measured within the $3 \mathrm{mJy} /$ beam contour) are presented in Table $1^{2}$.

Using the source identification scheme presented in Appendix A of Peretto \& Fuller (2009) we further identified 18 compact sources sitting within the filaments. The observed properties of these sources are given in Table 2. A number of sources have sizes that are close to, or even smaller than, the beam size (all sources with dashes in Table 2 are in this situation). These sources have significant emission peaks $(>3 \sigma)$, but because they are blended with nearby sources, their measured sizes appear smaller than the beam, and their properties are very uncertain. All these sources are confirmed with a higher resolution extinction map and SABOCA data (not shown here). The flux uncertainties in Table 1 reflect the difficulty of estimating the separation between the sources and the underlying filament. It is calculated by taking the average of the clipping and the bijection schemes of the flux estimates (Rosolowsky et al. 2008). We characterised the compact sources further by checking their association with Spitzer $24 \mu \mathrm{m}$ point-like sources, and their fragmentation as seen in the $8 \mu$ m extinction map (see Fig. 1 from Peretto \& Fuller 2010). We find that most of the sources are starless (13 out of 18) and that only MM10 appears to be sub-fragmented in extinction. The results of this analysis are also quoted in Table 2.

Assuming that the dust emission is optically thin at $1.2 \mathrm{~mm}$, the measured fluxes provide a direct measurement of the source mass. In Tables 3 and 4 we give the masses of all structures estimated using a specific dust opacity at $1.2 \mathrm{~mm} \kappa_{1.2} \mathrm{~mm}=$ $0.005 \mathrm{~cm}^{2} \mathrm{~g}^{-1}$, and dust temperatures of $12 \mathrm{~K}$ for filaments and starless sources, and $15 \mathrm{~K}$ for protostellar ones (Peretto et al. 2010). We also provide their volume densities, and the mass per unit length of filaments. All uncertainties result from propagation of the flux uncertainties. They do not take systematic uncertainties on the dust temperature/opacity into account,

\footnotetext{
2 The sizes are estimated in the same way as for the IRDC catalogue of Peretto \& Fuller (2009, see Appendix A), meaning that we calculate the matrix of moment of inertia of the pixels within the $3 \mathrm{mJy} / \mathrm{beam}$ contour for each filament. Then we diagonalise the matrix and get two values corresponding to the sizes along the major and minor axes of the filaments. The radius $R_{\text {eff }}$ corresponds to the radius of the disc having the same area as the filament. The $3 \mathrm{mJy} /$ beam contour encompasses all filaments, so we artificially divided the MAMBO emission at the filaments junction.
}

increasing the uncertainty on all calculated properties by an extra factor of $\leq 2$.

\subsection{Dense gas projected velocity field}

The $\mathrm{N}_{2} \mathrm{H}^{+}(1-0)$ spectra observed along the SDC13 filaments are displayed in Figs. 2 and 3. All spectra show strong detections $(S / N \geq 4)$ for all hyperfine line components and symmetric line profiles. Based on these observations, we estimated the systemic velocity of SDC13 at $+37 \mathrm{~km} \mathrm{~s}^{-1}$ (near position 8 in Fig. 2). From position 18 to 24 and 28 to 31 , we observe a secondary velocity component separated by $+17 \mathrm{~km} \mathrm{~s}^{-1}$. In Fig. 2 it can be identified as a small bump at $V \simeq+54 \mathrm{~km} \mathrm{~s}^{-1}$ on spectrum \#24. This higher velocity component most likely belongs to another cloud on the eastern side of SDC13 that overlaps, in projection, with the Fi-NE filament. For the remainder of the paper have we decided to ignore this higher velocity component.

We used the hyperfine structure line fitting routine of the CLASS software to derive line parameters, such as the centroid gas velocity and gas velocity dispersion (see Fig. 2). We represent the results from this fitting procedure in Fig. 4 . The colour and size of the circular symbols code the line-of-sight velocity and linewidth, respectively, of the gas as measured in $\mathrm{N}_{2} \mathrm{H}^{+}(1-0)$. The exact values are given in Table 5 for each position. Figure 4 shows that both the dense gas velocity and velocity dispersion vary smoothly along each filament, from one end to the other. The two quantities are strongly correlated. Along Fi-NE, we can actually see that the smooth variation in the velocity reaches a minimum (around position 25) to start increasing again towards position 31 . We believe that this shows that the northern and southern parts of Fi-NE are dynamically distinct. This is why we decided to subdivide this filament in two, Fi-NEn and Fi-NEs. The Fi-NE filaments are blue-shifted with respect to the systemic velocity of SDC13 while the Fi-NW and Fi-SE filaments are red-shifted. The filaments spatially and dynamically converge near position 8 at the systemic velocity of the system, i.e. $+37 \mathrm{~km} \mathrm{~s}^{-1}$. At this same position, the linewidth reaches a maximum of $\sim 2 \mathrm{~km} \mathrm{~s}^{-1}$, while at the filament ends the linewidth is the narrowest, i.e. $\sim 0.7 \mathrm{~km} \mathrm{~s}^{-1}$.

\section{Discussion}

\subsection{Timescales and filament stability}

The Spitzer protostellar objects that we identify in SDC13 filaments are typically embedded Class I (or young Class II) objects (see Appendix B). The age $\tau_{\mathrm{CI}}$ of such objects from prestellar core stage to Class I protostellar stage is $1 \mathrm{Myr} \leq$ $\tau_{\mathrm{CI}} \leq 2 \operatorname{Myr}$ (e.g. Kirk et al. 2005; Evans et al. 2009), consequently setting a lower limit of $1 \mathrm{Myr}$ for the age of the filaments themselves. Their widths ${ }^{3}$ as measured on the MAMBO map are $\sim 0.3$ pc. Taking a $3 \mathrm{D}$ velocity dispersion $\sigma_{3 \mathrm{D}}=\sqrt{3} \sigma_{\text {tot }}=1.0( \pm 0.3) \mathrm{km} \mathrm{s}^{-1}$, we obtain a crossing time $t_{\text {cross }}=0.3( \pm 0.1)$ Myr. Given their lower age limit of $1 \mathrm{Myr}$, the SDC13 filaments must therefore either be gravitationally bound or confined by ram/magnetic pressure. The thermal critical mass-per-unit-length for a $12 \mathrm{~K}$ isothermal filament is $M_{\text {line,crit }}^{\text {th }}=19 M_{\odot} / \mathrm{pc}$, and the observed values for the SDC13 filaments are higher by a factor of 4 to 8 (cf. Table 3),

\footnotetext{
3 These widths are compatible with the standard 0.1 pc FWHM width observed towards nearby interstellar filaments (Arzoumanian et al. 2011) since the SDC13 measurements we provide here extend beyond the FWHM of the filament profiles.
} 
Table 2. Observed properties of SDC13 cores.

\begin{tabular}{|c|c|c|c|c|c|c|c|c|c|c|}
\hline Name & $\begin{array}{c}\text { RA } \\
(\mathrm{J} 2000)\end{array}$ & $\begin{array}{c}\text { Dec } \\
(\mathrm{J} 2000)\end{array}$ & $\begin{array}{c}F_{1.2 \mathrm{~mm}}^{\mathrm{pk}} \\
(\mathrm{mJy} / \mathrm{beam})\end{array}$ & $\begin{array}{l}F_{1.2 \mathrm{~mm}}^{\mathrm{int}} \\
(\mathrm{mJy})\end{array}$ & $\begin{array}{c}\text { Maj. } \\
\left({ }^{\prime \prime}\right)\end{array}$ & $\begin{array}{c}\text { Min. } \\
\left({ }^{\prime \prime}\right)\end{array}$ & $\begin{array}{l}\text { PA } \\
\left({ }^{\circ}\right)\end{array}$ & $\begin{array}{l}R_{\text {eff }} \\
\left({ }^{\prime \prime}\right)\end{array}$ & Protostellar? & Fragmented? \\
\hline MM1 & $18: 14: 30.86$ & $-17: 33: 20.4$ & 51.8 & $94.5 \pm 34.3$ & 28.5 & 11.6 & +27 & 15.7 & yes & - \\
\hline MM2 & 18:14:28.53 & $-17: 33: 30.9$ & 48.3 & $73.2 \pm 23.3$ & 14.0 & 11.9 & +21 & 12.8 & no & no \\
\hline MM3 & $18: 14: 35.53$ & $-17: 30: 53.4$ & 34.6 & $22.3 \pm 16.5$ & 11.3 & 6.4 & +26 & 7.7 & no & no \\
\hline MM4 & 18:14:30.63 & $-17: 33: 59.0$ & 31.2 & $23.0 \pm 13.8$ & 10.5 & 6.8 & -49 & 8.4 & yes & - \\
\hline MM5 & 18:14:33.89 & $-17: 31: 14.4$ & 28.6 & $10.3 \pm 8.4$ & 8.4 & 3.8 & +39 & 5.6 & yes & no \\
\hline MM6 & $18: 14: 35.30$ & $-17: 30: 36.0$ & 28.4 & $8.8 \pm 7.7$ & 6.5 & 3.7 & -45 & 5.2 & no & no \\
\hline MM7 & $18: 14: 32.96$ & $-17: 34: 19.9$ & 26.3 & $26.0 \pm 17.6$ & 15.6 & 7.2 & -60 & 9.9 & yes & no \\
\hline MM8 & $18: 14: 29.93$ & $-17: 33: 48.4$ & 20.4 & - & - & - & - & - & no & - \\
\hline MM9 & $18: 14: 32.73$ & $-17: 31: 39.0$ & 19.9 & $9.8 \pm 7.0$ & 7.7 & 5.6 & +43 & 6.6 & no & no \\
\hline MM10 & $18: 14: 32.26$ & $-17: 32: 24.5$ & 18.8 & $31.0 \pm 17.3$ & 18.7 & 10.5 & +27 & 13.0 & no & yes \\
\hline MM11 & $18: 14: 27.37$ & $-17: 32: 42.0$ & 17.9 & $5.7 \pm 4.0$ & 7.00 & 3.9 & -32 & 5.2 & no & no \\
\hline MM12 & $18: 14: 34.59$ & $-17: 30: 21.9$ & 17.9 & - & - & - & - & - & no & - \\
\hline MM13 & $18: 14: 25.72$ & $-17: 32: 07.0$ & 17.8 & $6.5 \pm 4.2$ & 6.1 & 4.8 & -44 & 5.6 & no & no \\
\hline MM14 & $18: 14: 34.83$ & $-17: 34: 41.0$ & 17.7 & $14.0 \pm 11.0$ & 10.5 & 8.2 & -9 & 8.6 & no & - \\
\hline MM15 & $18: 14: 26.67$ & $-17: 32: 27.9$ & 16.2 & - & - & - & - & - & no & no \\
\hline MM16 & $18: 14: 24.56$ & $-17: 31: 42.4$ & 16.1 & $8.5 \pm 5.6$ & 12.2 & 4.1 & -35 & 6.8 & no & no \\
\hline MM17 & $18: 14: 24.79$ & $-17: 31: 56.4$ & 14.6 & - & - & - & - & - & no & no \\
\hline MM18 & $18: 14: 29.46$ & $-17: 33: 13.4$ & 13.8 & - & - & - & - & - & yes & - \\
\hline
\end{tabular}

Notes. Column 1: source name; Cols. 2 and 3: source coordinates; Col. 4: $1.2 \mathrm{~mm}$ dust continuum peak flux density; Col. 5: source 1.2 mm dust continuum integrated flux density. A dash indicates that the source is too weak/blended to extract any robust physical parameter; Col. 6: source major axis, angular size uncertainties are $\pm 1^{\prime \prime}$; Col. 7: source minor axis; Col. 8: source position angle; Col. 9: source geometric radius; Col. 9: tag informing on the protostellar/starless nature of the source; Col. 10: tag informing if a source is sub-fragmented on the $8 \mu \mathrm{m}$ extinction map. A dash indicates that the source is not seen in extinction.

Table 3. Physical properties of SDC13 filaments.

\begin{tabular}{lcccccc}
\hline \hline Name & $\begin{array}{c}\text { Sizes }_{\text {dec }} \\
(\mathrm{pc} \times \mathrm{pc})\end{array}$ & Aspect ratio & $\begin{array}{c}\text { Mass } \\
\left(M_{\odot}\right)\end{array}$ & $\begin{array}{c}\text { Density } \\
\left(\times 10^{4} \mathrm{~cm}^{-3}\right)\end{array}$ & $\begin{array}{c}M_{\text {line }} \\
\left(M_{\odot} \mathrm{pc}^{-1}\right)\end{array}$ & $\begin{array}{c}\nabla V \\
\left(\mathrm{~km} \mathrm{~s}^{-1} \mathrm{pc}^{-1}\right)\end{array}$ \\
\hline Fil-NEn & $1.8 \times 0.3$ & 6 & $256 \pm 32$ & $3.5 \pm 0.4$ & $142 \pm 18$ & $0.22 \pm 0.07$ \\
Fil-NEs & $1.6 \times 0.3$ & 5 & $211 \pm 30$ & $3.2 \pm 0.5$ & $117 \pm 19$ & $0.63 \pm 0.19$ \\
Fil-NW & $2.6 \times 0.2$ & 13 & $195 \pm 31$ & $4.1 \pm 0.7$ & $75 \pm 12$ & $0.36 \pm 0.11$ \\
Fil-SE & $1.9 \times 0.3$ & 6 & $216 \pm 32$ & $2.8 \pm 0.4$ & $114 \pm 17$ & $0.62 \pm 0.14$ \\
\hline
\end{tabular}

Notes. Column 1: filament name; Col. 2: deconvolved filament sizes; Col. 3: filament aspect ratios. Given the dynamics of the SDC13 filaments, i.e. all collapsing towards one of their end, the values given in this column correspond to half of the aspect ratios used for the modelling in Sect. 4.3; Col. 4: filament mass; Col. 5: filament volume density assuming cylindrical geometry; Col. 6: filament mass-per-unit-length; Col. 7: filament velocity gradients measured up to a distance of $1.5 \mathrm{pc}$ for all filaments (leaving out the flat part of the velocity profile, see Fig. 5).

Table 4. Physical properties of SDC13 cores.

\begin{tabular}{lccc}
\hline \hline Name & $\begin{array}{c}R_{\text {eff }}^{\text {dec }} \\
(\mathrm{pc})\end{array}$ & $\begin{array}{c}\text { Mass } \\
\left(M_{\odot}\right)\end{array}$ & $\begin{array}{c}\text { Density } \\
\left(\times 10^{4} \mathrm{~cm}^{-3}\right)\end{array}$ \\
\hline MM1 & 0.26 & $74.8 \pm 27.1$ & $1.8 \pm 0.6$ \\
MM2 & 0.21 & $81.1 \pm 25.8$ & $3.6 \pm 1.1$ \\
MM3 & 0.10 & $24.7 \pm 18.3$ & $10.2 \pm 7.6$ \\
MM4 & 0.11 & $18.1 \pm 10.9$ & $5.6 \pm 3.4$ \\
MM5 & $<0.05$ & $8.2 \pm 6.6$ & $>27.2 \pm 21.9$ \\
MM6 & $<0.05$ & $9.7 \pm 8.5$ & $>32.1 \pm 28.2$ \\
MM7 & 0.15 & $20.2 \pm 13.9$ & $2.5 \pm 1.7$ \\
MM8 & - & - & - \\
MM9 & 0.06 & $10.9 \pm 7.7$ & $20.9 \pm 14.8$ \\
MM10 & 0.21 & $34.3 \pm 19.2$ & $1.5 \pm 0.9$ \\
MM11 & $<0.05$ & $6.3 \pm 4.4$ & $>20.9 \pm 14.6$ \\
MM12 & - & - & - \\
MM13 & $<0.05$ & $7.3 \pm 4.7$ & $>24.2 \pm 15.6$ \\
MM14 & 0.12 & $15.6 \pm 12.2$ & $3.8 \pm 2.9$ \\
MM15 & - & - & - \\
MM16 & 0.07 & $9.4 \pm 6.1$ & $11.3 \pm 7.4$ \\
MM17 & - & - & - \\
MM18 & - & - & - \\
\hline
\end{tabular}

Notes. Column 1: source name; Col. 2: source deconvolved geometric radius; Col. 3: source mass; Col. 4: source volume density estimated assuming spherical geometry. meaning that they are thermally supercritical. However, if we consider non-thermal motions as an extra support against gravity, the picture slightly changes. We define the effective critical mass-per-unit-length as $M_{\text {line,crit }}^{\text {eff }}=2{\overline{\sigma_{\text {tot }}}}^{2} / G$, where $\overline{\sigma_{\text {tot }}}$ corresponds to the average total velocity dispersion along the filaments. Taking $\overline{\sigma_{\text {tot }}}=0.6 \mathrm{~km} \mathrm{~s}^{-1}$ (cf. Table 5), we obtain $1 \leq \frac{M_{\text {line,crit }}^{\text {eff }}}{M_{\text {line }}} \leq 2$ for all filaments, suggesting that they are critical. This ratio is probably slightly overestimated since the contribution from the longitudinal collapse of filaments (see following sections) probably contributes to the observed line widths in the form of non-supportive bulk motions. The SDC13 filaments match the relationship between $M_{\text {line }}$ and $M_{\text {line,crit }}^{\text {eff }}$ found by Arzoumanian et al. (2013) for thermally supercritical filaments.

\subsection{Dynamical evolution scenarios}

The smooth velocity structure observed along the SDC13 filaments shows that these filaments are dynamically coherent. The number of physical processes leading to a well organised parsec-scale velocity pattern, as observed in SDC13, is rather limited. Collapse, expansion, rotation, soft filament collision, and wind-driven acceleration are the five main possibilities. Rotation would imply that the SDC13 filaments rotate about an 
Table 5. $\mathrm{N}_{2} \mathrm{H}^{+}(1-0)$ spectra properties.

\begin{tabular}{lcccccc}
\hline \hline Id & $\begin{array}{c}\text { RA } \\
(\text { J2000 })\end{array}$ & $\begin{array}{c}\text { Dec } \\
(\mathrm{J} 2000)\end{array}$ & $\begin{array}{c}\text { Velocity } \\
\left(\mathrm{km} \mathrm{s}^{-1}\right)\end{array}$ & $\begin{array}{c}F W H M \\
\left(\mathrm{~km} \mathrm{~s}^{-1}\right)\end{array}$ & $\begin{array}{c}\sigma_{\mathrm{NT}} \\
\mathrm{km} \mathrm{s}^{-1}\end{array}$ & $\begin{array}{c}\sigma_{\text {tot }} \\
\mathrm{km} \mathrm{s}^{-1}\end{array}$ \\
\hline P1 & $18: 14: 35.29$ & $-17: 34: 41.9$ & 37.83 & 0.87 & 0.37 & 0.42 \\
P2 & $18: 14: 34.50$ & $-17: 34: 33.7$ & 37.89 & 0.95 & 0.40 & 0.45 \\
P3 & $18: 14: 33.77$ & $-17: 34: 25.8$ & 37.88 & 1.32 & 0.56 & 0.60 \\
P4 & $18: 14: 32.95$ & $-17: 34: 18.0$ & 37.68 & 1.58 & 0.67 & 0.70 \\
P5 & $18: 14: 32.16$ & $-17: 34: 10.3$ & 37.58 & 1.60 & 0.68 & 0.71 \\
P6 & $18: 14: 31.36$ & $-17: 34: 02.8$ & 37.38 & 1.57 & 0.67 & 0.70 \\
P7 & $18: 14: 30.54$ & $-17: 33: 58.1$ & 37.25 & 1.76 & 0.75 & 0.78 \\
P8 & $18: 14: 29.69$ & $-17: 33: 49.4$ & 37.09 & 1.87 & 0.79 & 0.82 \\
P9 & $18: 14: 28.53$ & $-17: 33: 38.0$ & 37.19 & 1.92 & 0.81 & 0.84 \\
P10 & $18: 14: 28.11$ & $-17: 33: 25.8$ & 37.24 & 1.97 & 0.84 & 0.86 \\
P11 & $18: 14: 27.84$ & $-17: 33: 12.7$ & 37.48 & 1.50 & 0.64 & 0.67 \\
P12 & $18: 14: 27.50$ & $-17: 32: 59.9$ & 37.41 & 1.22 & 0.52 & 0.56 \\
P13 & $18: 14: 27.29$ & $-17: 32: 46.1$ & 37.49 & 1.03 & 0.43 & 0.48 \\
P14 & $18: 14: 26.71$ & $-17: 32: 35.2$ & 37.60 & 1.03 & 0.43 & 0.48 \\
P15 & $18: 14: 25.34$ & $-17: 32: 15.0$ & 37.39 & 0.68 & 0.28 & 0.35 \\
P16 & $18: 14: 30.74$ & $-17: 33: 38.2$ & 36.83 & 1.72 & 0.73 & 0.76 \\
P17 & $18: 14: 29.58$ & $-17: 33: 32.2$ & 36.96 & 1.86 & 0.79 & 0.82 \\
P18 & $18: 14: 30.83$ & $-17: 33: 18.7$ & 36.82 & 1.85 & 0.78 & 0.81 \\
P19 & $18: 14: 31.09$ & $-17: 33: 05.9$ & 36.59 & 1.52 & 0.64 & 0.67 \\
P20 & $18: 14: 31.36$ & $-17: 32: 52.8$ & 36.48 & 1.35 & 0.57 & 0.61 \\
P21 & $18: 14: 31.67$ & $-17: 32: 39.7$ & 36.33 & 0.88 & 0.37 & 0.42 \\
P22 & $18: 14: 32.01$ & $-17: 32: 26.9$ & 36.19 & 1.06 & 0.45 & 0.49 \\
P23 & $18: 14: 32.17$ & $-17: 32: 13.1$ & 36.21 & 1.01 & 0.43 & 0.48 \\
P24 & $18: 14: 32.17$ & $-17: 31: 59.6$ & 36.17 & 0.87 & 0.37 & 0.42 \\
P25 & $18: 14: 32.17$ & $-17: 31: 45.7$ & 36.18 & 0.66 & 0.27 & 0.34 \\
P26 & $18: 14: 32.38$ & $-17: 31: 32.6$ & 36.19 & 0.66 & 0.27 & 0.34 \\
P27 & $18: 14: 32.93$ & $-17: 31: 20.6$ & 36.34 & 0.79 & 0.33 & 0.39 \\
P28 & $18: 14: 33.61$ & $-17: 31: 10.8$ & 36.37 & 1.01 & 0.43 & 0.48 \\
P29 & $18: 14: 34.47$ & $-17: 31: 04.4$ & 36.44 & 1.29 & 0.55 & 0.59 \\
P30 & $18: 14: 35.23$ & $-17: 30: 56.2$ & 36.44 & 1.38 & 0.58 & 0.62 \\
P31 & $18: 14: 35.65$ & $-17: 30: 43.1$ & 36.47 & 1.44 & 0.61 & 0.64 \\
\hline & & & & & & \\
\hline
\end{tabular}

Notes. The uncertainties on all velocity measurements are $\pm 0.05 \mathrm{~km} \mathrm{~s}^{-1}$. Column 1: spectrum position number (as displayed in Figs. 2 and 3); Cols. 2 and 3: position coordinates; Col. 4: spectrum best-fit velocity; Col. 5: spectrum best-fit line width; Col. 6: spectrum non-thermal velocity dispersion component; Col. 7: spectrum total (thermal+non-thermal) velocity dispersion. We assumed a gas temperature of $12 \mathrm{~K}$ for these calculations.

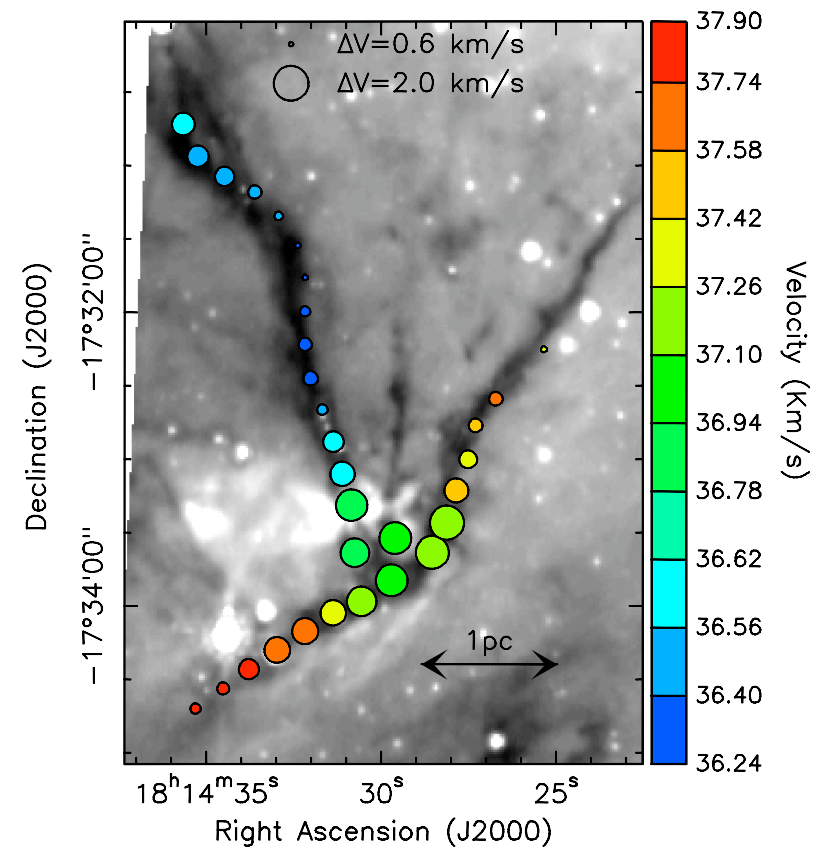

Fig. 4. Spitzer $8 \mu \mathrm{m}$ image of SDC13 in grey scale on top of which we symbolised the results of the $\mathrm{N}_{2} \mathrm{H}^{+}(1-0)$ HFS fitting as circles. The colour of the symbols indicate the gas velocity while their sizes indicate the gas velocity dispersion (FWHM). axis going through the filament junction. The resulting geometry is unrealistic in the context of interstellar cloud dynamics, because differential rotation would tear apart each filament in a crossing time $t_{\text {cross }} \simeq 0.3 \mathrm{Myr}$ (cf. Sect. 4.1). No expanding source of energy (such as HII region or cluster of outflows ${ }^{4}$ ) is located at the centre of SDC13, rendering the expansion scenario unlikely, while colliding filaments cannot account for the velocity pattern observed along Fi-NEn. Finally, wind-driven acceleration powered by a nearby stellar cluster is a credible scenario. In particular, as we can see in Fig. 1 a mid-infrared nebulosity, along with a number of infrared compact sources, are observed east of MM1, probably interacting with it and affecting its velocity through expanding winds. However, it probably does not affect the dynamics of the entire network of filaments. Similar to the colliding flow scenario, the wind-driven scenario can hardly explain the smooth velocity field observed in these filaments and, in particular, the one along Fi-NEn.

We therefore consider that longitudinal collapse is the best option to explain the velocity structure observed in SDC13. Gravity can naturally account for a velocity gradient along the filaments as the gas collapses towards the centre of the system. In this context, one can argue that there is a primary gravitational potential well centre near position 8 , towards which all the gas is

\footnotetext{
4 We observed few core positions in $\mathrm{SiO}(2-1)$, well known tracer of protostellar outflows, but did not get any wing emission.
} 

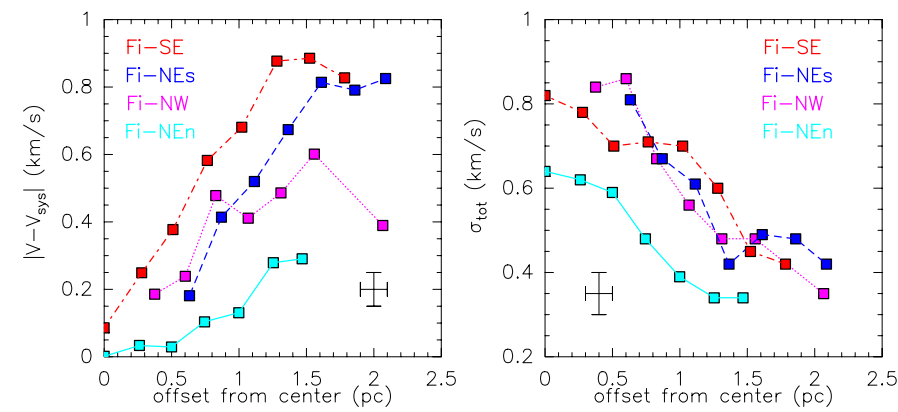

Fig. 5. Left: line-of-sight velocity profile of the dense gas within each filament as a function of position form the well centres, i.e. position 8 for Fi-NW (purple dotted line), Fi-SE (red dashed-dotted line), Fi-NEs (blue dashed line), and position 31 for Fi-NEn (cyan solid line). Right: same as left panel but for the total (thermal+non-thermal) dense gas velocity dispersion.

collapsing, and a secondary one near position 31 towards which only Fi-NEn is infalling. In this collapse scenario, the red-shifted filaments, i.e. Fi-NW and Fi-SE, are in the foreground with respect to the primary centre of the system, while the blue-shifted ones, Fi-NEs and Fi-NEn, are in the background. The dense gas velocity observed along the filaments is therefore interpreted as a projected infall velocity profile. Figure 5 displays these profiles for all four filaments. We can see that for each filament the velocity is a linear function of the distance to the centre (gradients are given in Table 3 ) up to $\sim 1.5 \mathrm{pc}$ and then flattens out. This is expected when homologous free-fall collapse of filament have centrally concentrated density profiles along their crest (see Fig. 6 from Pon et al. 2011; Fig. 5 from Peretto et al. 2007; and Fig. 1 from Myers 2005).

In the context of longitudinal collapse, the observed velocity gradients are an indication of the time evolution of each filament as the gradient becomes steeper during collapse. We can therefore potentially constrain the age of a filament by measuring its velocity gradient. Pon et al. (2012) showed that the collapse time of free-falling filaments is $\tau_{1 \mathrm{D}}=\sqrt{2 / 3} A \tau_{3 \mathrm{D}}$ where $\tau_{3 \mathrm{D}}$ is the spherical free fall time at the same volume density, and $A$ is the aspect ratio of the filament. This equation shows that the collapsing time of a filament can be significantly longer than the standard $\tau_{3 \mathrm{D}}$. One can compute the time evolution of the filament velocity gradient as a function of the semi-major axis $Z$, and this for a given initial density $n_{0}$, initial aspect ratio $A_{0}$, and initial semi-major axis $Z_{0}$, and then compare these to the SDC13 filament observed values (see Appendix A for more details). Figure 6 shows that the velocity gradients of modelled filaments are consistent with the observed ones after $1 \mathrm{Myr}$ to $4 \mathrm{Myr}$ of evolution, depending on the projection angles. Similar collapsing timescales have recently been reported for the massive-star forming filament NGC 6334 (Zernickel et al. 2013). Also, that Fi-NEn seems dynamically younger is consistent with the idea that it is collapsing towards a secondary potential well centre around position 31. Despite the limitations of this comparison (SDC13 is more of a hub-filament system rather than a single filament - cf. Fig. 7), these timescales are consistent with the presence of Class I protostellar sources in the SDC13 filaments.

\subsection{Gravity-driven turbulence and core formation}

Figure 5 (right) shows the total velocity dispersion (including thermal and non-thermal motions - cf. Arzoumanian et al. 2013) along each filaments. We can see a strong correlation between

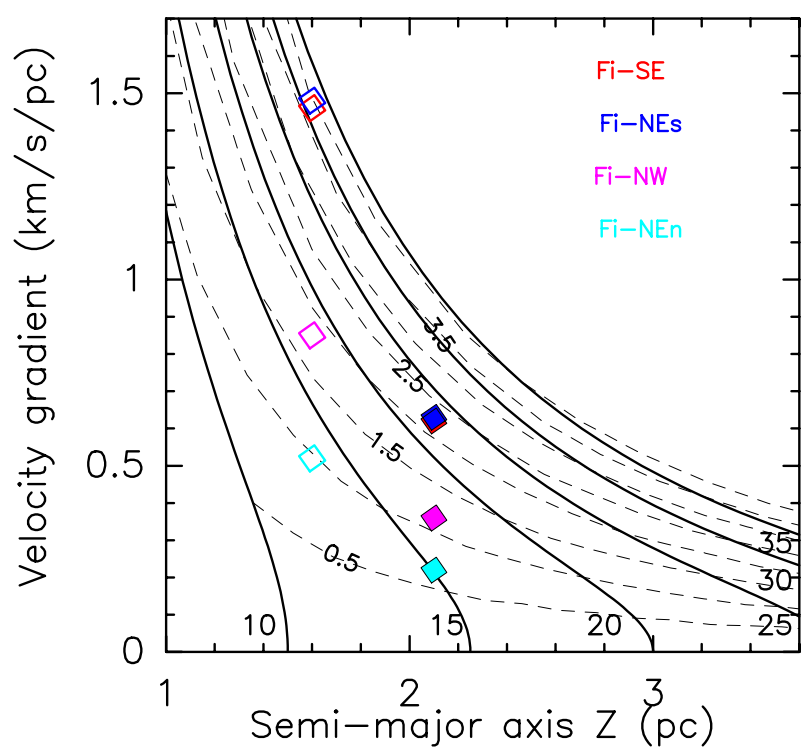

Fig. 6. Time evolution of free-falling filaments in the $Z-\nabla V$ parameter space. All filaments have the same initial density $n_{0}=4 \times 10^{4} \mathrm{~cm}^{-3}$. Each solid line represents the evolution of a filament with a different initial aspect ratio $A_{0}$ indicated at the bottom end. Dashed lines represent isochrones from $0.5 \mathrm{Myr}$ to $4 \mathrm{Myr}$ separated by $0.5 \mathrm{Myr}$. The solid and empty squares mark the positions of the four filaments, for projection angles of $45^{\circ}$ and $67^{\circ}$, respectively (cf. text and Appendix A).

this quantity and the dense gas velocity, the velocity dispersion increasing towards each of the two centres of collapse (positions 8 and 31). During the collapse of the filaments, the gravitational energy of the gas is converted into kinetic energy (e.g. Peretto et al. 2007; Vázquez-Semadeni et al. 2007). This has been proposed as the main process by which radially collapsing filaments manage to keep a constant width (Arzoumanian et al. 2013). The velocity dispersion resulting from gravitational energy conversion is then a function of the infall velocity and the gas density (Klessen \& Hennebelle 2010). The global dynamical evolution of a supercritical filament initially at rest, with uniform density, can be described in two stages (e.g. Peretto et al. 2007). In the first stage, the gas at the filament ends is accelerated more efficiently, and the filament develops a linear velocity gradient increasing from centre to ends. In the second stage, as the matter is accumulated at the centre, the velocity gradient starts to reverse owing to the acceleration close to the central mass becoming larger than at the filament ends. It is clear that in the second stage, both the infall velocity and the velocity dispersion will increase faster at the centre than at the filament ends. However, in the first stage we might expect the infall velocity dispersion and the infall velocity to be greater at the filament ends. In the case of SDC13, the infall velocity is higher at the filament ends, but the velocity dispersion is larger at the centre. This could be explained by the SDC13 filaments being in an intermediate evolutionary stage and/or the initial density profile of SDC13 not being uniform. In fact, SDC13 as a whole has a more complex geometry and density structure than the ones of a single filament, more like a hub-filament system (Myers 2009). In such a configuration, the potential well is dominated by the central mass of the hub. The resulting hub density profile might then favour an early increase in the velocity dispersion during the hub collapse. However, this is speculation and remains to be tested.

The MM1 and MM2 sources, the most massive ones in SDC13, are located near the centre of the system. Within the context of the free-fall collapse scenario, these sources must 


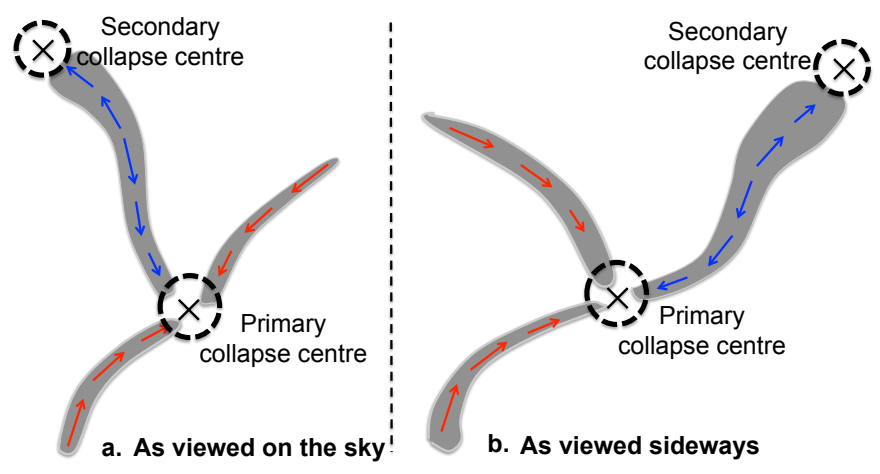

Fig. 7. Schematic representation of the the SDC13 velocity field (arrows) a) as viewed on the plane of the sky; $\mathbf{b}$ ) as viewed sideways with the observer on the left-hand-side of the plot. The two centres of collapse are symbolised by black dashed circles and a cross at their centres.

have formed within a tenth of a pc from their current position, suggesting that MM1 and MM2 have basically formed in situ, near the converging point of the filaments. This location is privileged for source mass growth since flows of dense gas are running towards it, both bringing more material to be accreted and locally increasing the velocity dispersion. We can quantify what impact both processes have on core formation. The mass infall rate due to material running through the filaments can be estimated following $\dot{M}_{\text {inf }}=\pi(W / 2)^{2} \rho_{\text {fil }} V_{\text {inf }}$ where $W$ is the filament width, $\rho_{\text {fil }}$ the filament density, and $V_{\text {inf }}$ the infall velocity. Using the average values given in Table 3, along with an infall velocity $V_{\text {inf }} \simeq 0.2 \mathrm{~km} \mathrm{~s}^{-1}$ at the core locations, we find that $\dot{M}_{\text {inf }} \simeq$ $2.5 \times 10^{-5} M_{\odot} / y r$. This means that over a million years each filament would bring $\sim 25 M_{\odot}$ to the SDC13 centre. This is probably an upper limit since the gas did not fall in for a million years with its current infall velocity. Now, if we consider that the gravitydriven turbulence acts as a support against gravity, then the effective Jeans mass becomes $M_{J}^{\text {eff }}=0.9 M_{\odot}\left(\sigma_{\text {tot }} / 0.2 \mathrm{~km} \mathrm{~s}^{-1}\right)^{3}$. With a gas velocity dispersion $\sigma_{\text {tot }} \simeq 0.8 \mathrm{~km} \mathrm{~s}^{-1}$ towards the centre of the system, the effective Jeans mass goes up to $M_{J}^{\mathrm{eff}} \simeq 60 M_{\odot}$. This is close to the observed masses for MM1 and MM2, and it suggests that filament longitudinal collapse might lead to the formation of super-Jeans cores more as a result of enhanced turbulent support than as a result of large-scale accretion.

\section{Conclusion}

The presence of protostellar sources and organised velocity gradients along the SDC13 filaments suggest that local and largescale longitudinal collapse are taking place simultaneously. As a result of the high aspect ratios of the SDC13 filaments, the timescale of the former process is much shorter than for the latter. Here we propose that the large-scale longitudinal collapse mostly contributes to the increase in the turbulent support towards the centre of collapse where starless cores with masses that are an order of magnitude higher than the thermal Jeans mass can form. This study therefore suggests that super-Jeans prestellar cores may form at the centre of collapsing clouds. Despite this increase in support at the centre of SDC13, the MM1 and MM2 masses are still much lower than the mass of the O-type star-forming core sitting at the centre of SDC335 (Peretto et al. 2013). We speculate that the major difference resides in the mass and morphology of the surrounding gas reservoirs surrounding these sources. Dense, collapsing, spherical clouds of cold gas are more efficient in concentrating matter at their centres in a short amount of time. The study of a larger sample of massive star-forming clouds presenting all sort of morphologies is required to test this assertion.

Acknowledgements. We thank the anonymous referee whose report helped improve the quality of this paper. We would like to thank Alvaro Hacar for helping with the MAMBO data reduction. And finally, we want to thank the IRAM $30 \mathrm{~m}$ staff for their support during the observing runs. N.P. was partly supported by a CEA/Marie Curie Eurotalents fellowship and benefited from the support of the European Research Council advanced grant ORISTARS (Grant Agreement No. 291294).

\section{References}

Allen, L. E., Calvet, N., D'Alessio, P., et al. 2004, ApJS, 154, 363 André, P., Men'shchikov, A., Bontemps, S., et al. 2010, A\&A, 518, L102 Arzoumanian, D., André, P., Didelon, P., et al. 2011, A\&A, 529, L6 Arzoumanian, D., André, P., Peretto, N., \& Könyves, V. 2013, A\&A, 553, A119

Busquet, G., Zhang, Q., Palau, A., et al. 2013, ApJ, 764, L26

Carey, S. J., Noriega-Crespo, A., Mizuno, D. R., et al. 2009, PASP, 121, 76

Chapman, N. L., Mundy, L. G., Lai, S.-P., \& Evans, N. J. 2009, ApJ, 690, 496 Churchwell, E., Babler, B. L., Meade, M. R., et al. 2009, PASP, 121, 213 Diolaiti, E., Bendinelli, O., Bonaccini, D., et al. 2000, A\&AS, 147, 335 Duarte-Cabral, A., Fuller, G. A., Peretto, N., et al. 2010, A\&A, 519, A27 Dunham, M. M., Crapsi, A., Evans, II, N. J., et al. 2008, ApJS, 179, 249 Evans, N. J., Dunham, M. M., Jørgensen, J. K., et al. 2009, ApJS, 181, 321 Flaherty, K. M., Pipher, J. L., Megeath, S. T., et al. 2007, ApJ, 663, 1069 Hacar, A., \& Tafalla, M. 2011, A\&A, 533, A34

Henshaw, J. D., Caselli, P., Fontani, F., et al. 2013, MNRAS, 428, 3425 Kirk, J. M., Ward-Thompson, D., \& André, P. 2005, MNRAS, 360, 1506 Kirk, H., Myers, P. C., Bourke, T. L., et al. 2013, ApJ, 766, 115 Klessen, R. S., \& Hennebelle, P. 2010, A\&A, 520, A17

Lada, C. J., \& Wilking, B. A. 1984, ApJ, 287, 610

Megeath, S. T., Allen, L. E., Gutermuth, R. A., et al. 2004, ApJS, 154, 367 Miettinen, O. 2012, A\&A, 540, A104

Molinari, S., Swinyard, B., Bally, J., et al. 2010, A\&A, 518, L100

Myers, P. C. 2005, ApJ, 623, 280

Myers, P. C. 2009, ApJ, 700, 1609

Ostriker, J. 1964, ApJ, 140, 1056

Parsons, H., Thompson, M. A., \& Chrysostomou, A. 2009, MNRAS, 399, 1506 Peretto, N., \& Fuller, G. A. 2009, A\&A, 505, 405

Peretto, N., \& Fuller, G. A. 2010, ApJ, 723, 555

Peretto, N., Hennebelle, P., \& André, P. 2007, A\&A, 464, 983

Peretto, N., Fuller, G. A., Plume, R., et al. 2010, A\&A, 518, L98

Peretto, N., Fuller, G. A., Duarte-Cabral, A., et al. 2013, A\&A, 555, A112

Pon, A., Johnstone, D., \& Heitsch, F. 2011, ApJ, 740, 88

Pon, A., Toalá, J. A., Johnstone, D., et al. 2012, ApJ, 756, 145

Ragan, S. E., Heitsch, F., Bergin, E. A., \& Wilner, D. 2012, ApJ, 746, 174

Reid, M. J., Menten, K. M., Zheng, X. W., et al. 2009, ApJ, 700, 137

Rosolowsky, E. W., Pineda, J. E., Kauffmann, J., \& Goodman, A. A. 2008, ApJ, 679, 1338

Sadavoy, S. I., Di Francesco, J., \& Johnstone, D. 2010, ApJ, 718, L32

Vázquez-Semadeni, E., Gómez, G. C., Jappsen, A. K., et al. 2007, ApJ, 657, 870

Wilking, B. A., Lada, C. J., \& Young, E. T. 1989, ApJ, 340, 823

Zernickel, A., Schilke, P., \& Smith, R. J. 2013, A\&A, 554, L2

Pages 8 to 10 are available in the electronic edition of the journal at http://www. aanda.org 
A\&A 561, A83 (2014)

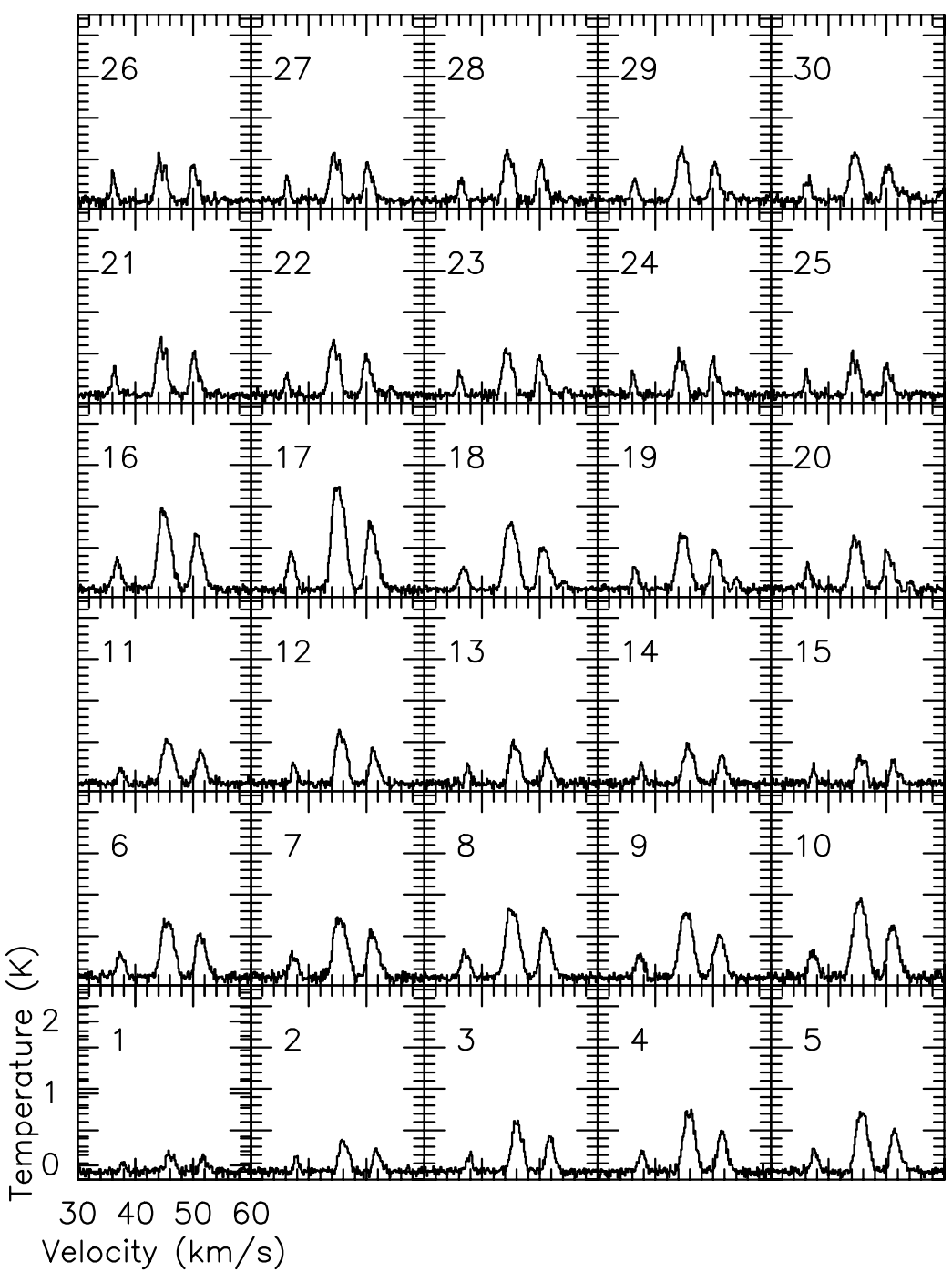

Fig. 3. $\mathrm{N}_{2} \mathrm{H}^{+}(1-0)$ spectra observed for each position along the SDC13 filaments (see Fig. 2). 


\section{Appendix A: Homologous free-fall collapse of filaments}

The equation of motion for a filament edge in homologous freefall collapse is given by (Pon et al. 2012)

$$
\frac{\mathrm{d}^{2} Z(t)}{\mathrm{d} t^{2}}=-\frac{G M}{Z(t)^{2}}
$$

where $Z(t)$ is the semi-major axis of the filament, and $M$ its mass. Multiplying each side of Eq. (A.1) by $\frac{\mathrm{d} Z(t)}{\mathrm{d} t}$ and integrating over $t$, we obtain

$$
\frac{\mathrm{d} Z(t)}{\mathrm{d} t}=V(t)=-\sqrt{2 G M\left[Z(t)^{-1}+\alpha\right]}
$$

where $\alpha$ is the constant of integration. Now, if we consider a filament initially at rest, we have $V(t=0)=0$, and therefore $\alpha=-Z_{0}^{-1}$, where $Z_{0}=Z(t=0)$ is the initial semi-major axis of the filament. We can solve for $Z(t)$ by setting $Z(t) / Z_{0}=\cos ^{2} \beta$ in Eq. (A.2) and integrating over $t$ again. We then obtain

$\beta+\frac{1}{2} \sin 2 \beta=t \sqrt{2 G M / Z_{0}^{3}}$

or, when replacing the mass by $M=2 \pi R^{2} Z_{0} \rho_{0}$,

$\beta+\frac{1}{2} \sin 2 \beta=\frac{t}{A_{0}} \sqrt{4 \pi G \rho_{0}}$

where $A_{0}$ is the initial aspect ratio of the filament and $\rho_{0}$ its initial density. The filament free-fall times $\tau_{1 \mathrm{D}}$ is calculated for $Z(t) / Z_{0}=0$, which means when $\beta=\pi / 2$. We therefore obtain

$\tau_{1 \mathrm{D}}=A_{0} \sqrt{\frac{\pi}{16 G \rho_{0}}}=\sqrt{2 / 3} A_{0} \tau_{3 \mathrm{D}}$

where $\tau_{3 D}$ is the spherical free-fall time at the same density. Finally, given that the velocity gradient is linear during the homologous collapse of a filament, we can compute the velocity gradient $\nabla V=V(t) / Z(t)$.

To compute the different values plotted in Fig. 6 we sampled $\beta$ between 0 and $\pi / 2$ and used the relevant equations presented in this appendix. We further assumed that the radius of the filaments stays the same during the collapse. For the purpose of the calculations, we took $R=0.15 \mathrm{pc}$. Figure 6 shows the evolution of seven filaments (solid lines) having all the same initial density $n_{0}=4 \times 10^{4} \mathrm{~cm}^{-3}$ but a different initial aspect ratio ${ }^{5}$.

Nearly all quantities presented in this paper are affected by projection effect. We can only correct for it if we know the projection angle $\theta$ of the filaments with respect to the line of sight. Unfortunately, it is impossible to know the exact value of $\theta$ for a given filament. However, for randomly sampled filament orientations one have $\langle\theta\rangle=67^{\circ}$. For this letter, we therefore considered two cases, the case where $\theta=45^{\circ}$ and the case where $\theta=67^{\circ}$. The corrected values for the velocity, semi-major axis, and velocity gradients are $V_{\text {corr }}=V_{\text {obs }} / \cos (\theta), L_{\text {corr }}=L_{\text {obs }} / \sin (\theta)$, and $\nabla V_{\text {corr }}=\nabla V_{\text {obs }} \tan (\theta)$. For $\theta=45^{\circ}$ these corrections meant that the observed velocity gradients remain unaffected, but the semi-major axis becomes larger by a factor 1.4. For $\theta=67^{\circ}$, the velocity gradient increases by a factor 2.4 and the semimajor axis by a factor 1.1. The points in Fig. 6 corresponding

\footnotetext{
5 Since we fixed the filament radius, $A_{0}$ and $Z_{0}$ are not independent parameters. Also, the aspect ratios given in Table 3 correspond to half of this $A_{0}$ parameter.
}

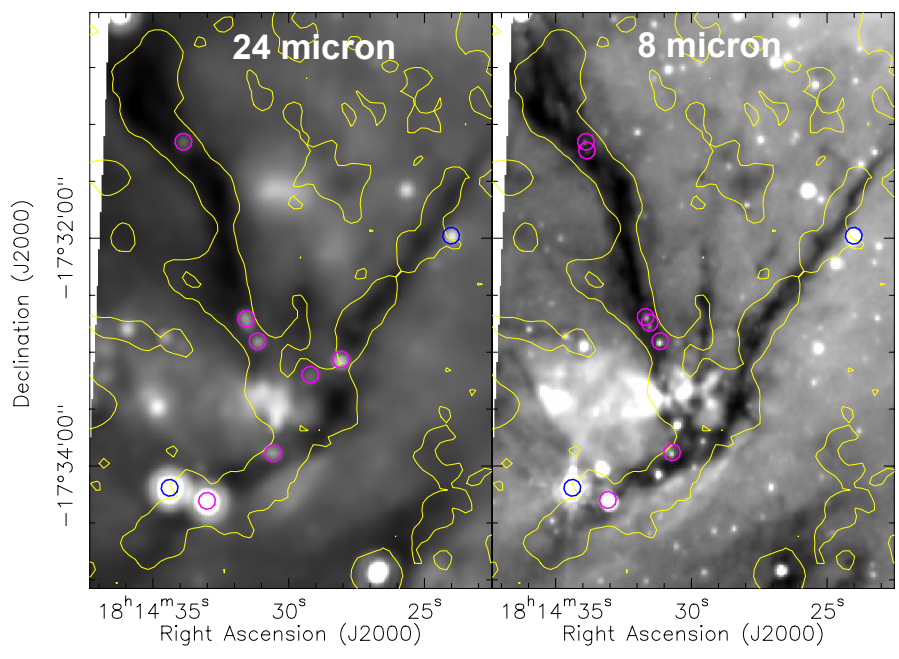

Fig. B.1. Left: Spitzer $24 \mu \mathrm{m}$ image of SDC13 (greyscale) on which we over-plotted the MAMBO $3 \mathrm{mJy} /$ beam contour (yellow). In this image we also report the positions of the seven protostellar sources identified along the SDC13 filaments (purple circles), along with the detection of two extra sources located on the edge of the outer edge of the contour (blue circles). Right: $8 \mu \mathrm{m}$ image of SDC13 (greyscale). The contour is the same as in the left panel. The purple circles mark the positions of the nine $8 \mu \mathrm{m}$ sources identified towards the identified $24 \mu \mathrm{m}$ sources. The meaning of the symbol's colour is the same as in the left panel.

to the four SDC13 filaments have been corrected by such factors. Much larger angles would lead to very large velocity gradients that have never been observed on parsec scales, while much smaller angles would imply very large initial aspect ratios and semi-major axes. Even though we cannot rule out such configurations, they are unlikely.

\section{Appendix B: Protostellar classes of SDC13 sources}

Infrared spectral indexes have been used for more than 20 years to classify YSOs according to their evolutionary stages. The idea is that the peak of a YSO spectral energy distribution (SED) evolves from submillimetre to infrared during the first few million years of its life. Calculating then the slope, i.e. the spectral index, of the SED at a critical frequency range allows the quantification of this evolution. YSOs classes are traditionally defined (e.g. Lada \& Wilking 1984; Wilking et al. 1989) between $2 \mu \mathrm{m}$ and $10 \mu \mathrm{m}$ by calculating the following quantity $\alpha_{[2-10]}=\frac{\mathrm{d}\left(\log \left(\lambda S_{\lambda}\right)\right)}{\mathrm{d}(\log (\lambda))}$, Class I sources having $\alpha_{[2-10]}>0$, Class II sources $0>\alpha_{[2-10]}>-1.6$, and Class III sources $\alpha_{[2-10]}<-1.6$.

In the context of this study we focussed on sources identified at $24 \mu \mathrm{m}$. We only considered sources that are spatially coincident with the SDC13 filaments (purple circles in Fig. B.1), limiting potential background/foreground contamination. To extract sources we used the Starfinder algorithm (Diolaiti et al. 2000) and found seven $24 \mu \mathrm{m}$ sources, five of which have $8 \mu \mathrm{m}$ counterparts $^{6}$ (see Fig. B.1). We then calculated their spectral index as estimated between $8 \mu \mathrm{m}$ and $24 \mu \mathrm{m}$ (fluxes of multiple $8 \mu \mathrm{m}$ sources have been combined, and for those with no $8 \mu \mathrm{m}$ counterpart, we used the $8 \mu \mathrm{m}$ detection limit of $2 \mathrm{mJy}$ ). One advantage provided by these two wavelengths is that they are similarly affected by extinction (Flaherty et al. 2007; Chapman et al. 2009), which implies that estimated values of $\alpha_{[8-24]}$ are not much affected by extinction. As a result, we find three sources

6 Note that two $24 \mu \mathrm{m}$ sources split up into two $8 \mu \mathrm{m}$ sources. 
with $-0.4<\alpha_{[8-24]}<0$, and another four with $0<\alpha_{[8-24]}<2.2$. Note that we also report the detection of two additional protostellar sources located on the outer edge of the filament (blue circles in Fig. B.1). Their spectral indexes are $\sim-1.5$, indicating that they might well be background/foreground sources. Note that we do not have any detection towards MM1 at $24 \mu \mathrm{m}$. This is because the $24 \mu \mathrm{m}$ emission is rather diffuse towards this source, and Starfinder failed to find any compact sources, a requirement for protostar detection.

We then used the YSO C2D database of Serpens, Ophiucchi, and Perseus (Evans et al. 2009) to compare the $\alpha_{[2-10]}$ spectral index with $\alpha_{[8-24]}$. The results of this comparison are displayed in Fig. B.2. Although there is some dispersion and not a one-to-one correlation, there is still a linear correlation between the two indexes. Despite the dispersion in $\alpha_{[8-24]}$, this plot strongly suggests that the seven protostellar sources identified within the SDC13 filaments have mid-infrared spectral indexes consistent with Class I and/or young Class II objects.

We further analysed the properties of the nine $8 \mu \mathrm{m}$ sources (see Fig. B.1) by constructing a colour-colour diagram using all IRAC Spitzer bands between $3.6 \mu \mathrm{m}$ and $8 \mu \mathrm{m}$. Depending on their colours, sources of different classes are expected to sit in different regions of such diagram (Allen et al. 2004; Megeath et al. 2004). In Fig. B.3 we can see that seven sources sit in the Class I region of the diagram, while the two remaining sources are clearly sitting in the Class III/stellar contamination region. The latter are in fact the two sources located on the edge of the filament (i.e. the blue circles in Fig. B.1), already identified as likely foreground/background sources from our spectral index analysis. Also, extinction can significantly affect the source location in this plot, mostly artificially increasing their [3.6]-[4.5] mag. However, the extracted sources are red enough that even for an extinction of $A_{v}=50$, most identified sources would remain in the Class I region of the diagram, the other ones just moving to the other side of the Class II/Class I border. This is consistent with the $\alpha_{[8-24]}$ analysis performed above.

Finally, based on the $24 \mu \mathrm{m}$ flux of protostellar sources and an assumed extinction, one may obtain an estimate of the source bolometric luminosity (Dunham et al. 2008). Following Parsons et al. (2009) we estimate that, with extinctions in the range of $10<A_{v}<50$ (matching the range of observed dust column density in SDC13) and $24 \mu \mathrm{m}$ fluxes between $10 \mathrm{mJy}$ and $60 \mathrm{mJy}$ for all but one source, the SDC13 protostellar sources have bolometric luminosities in the range of $10 L_{\odot}$ to few $100 L_{\odot}$. Such luminosities stand at the high end of the luminosity distribution of nearby low-mass protostars (Evans et al. 2009), possibly indicating a slightly shorter lifetime of the SDC13 protostellar sources compared to the standard Class I low-mass protostar lifetime.

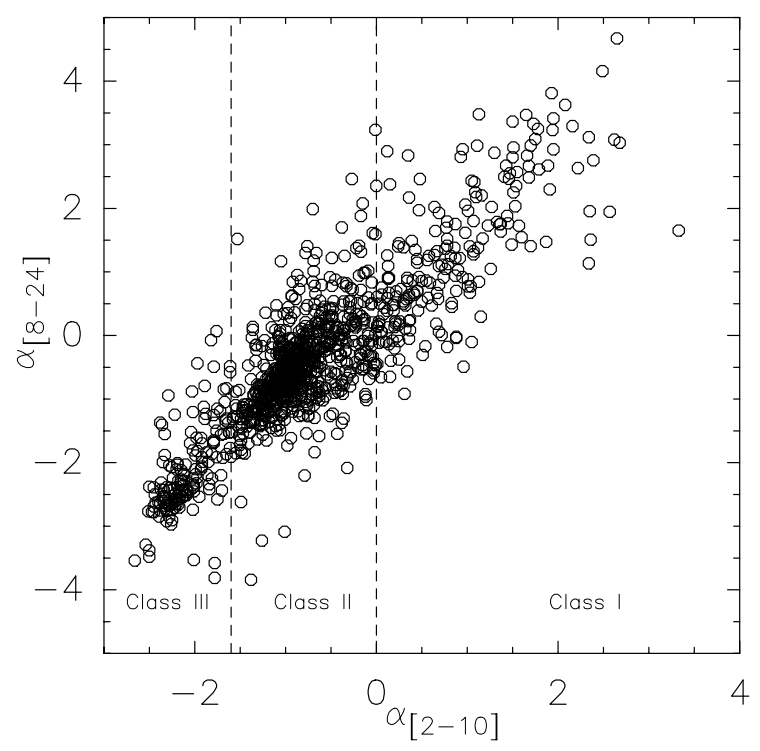

Fig. B.2. Mid-infrared spectral indexes for all $\sim 1000$ YSOs from the C2D catalogue (Evans et al. 2009). For each source the spectral index has been calculated for two wavelength ranges: from $2 \mu \mathrm{m}$ to $10 \mu \mathrm{m}$ and from $8 \mu \mathrm{m}$ to $24 \mu \mathrm{m}$.

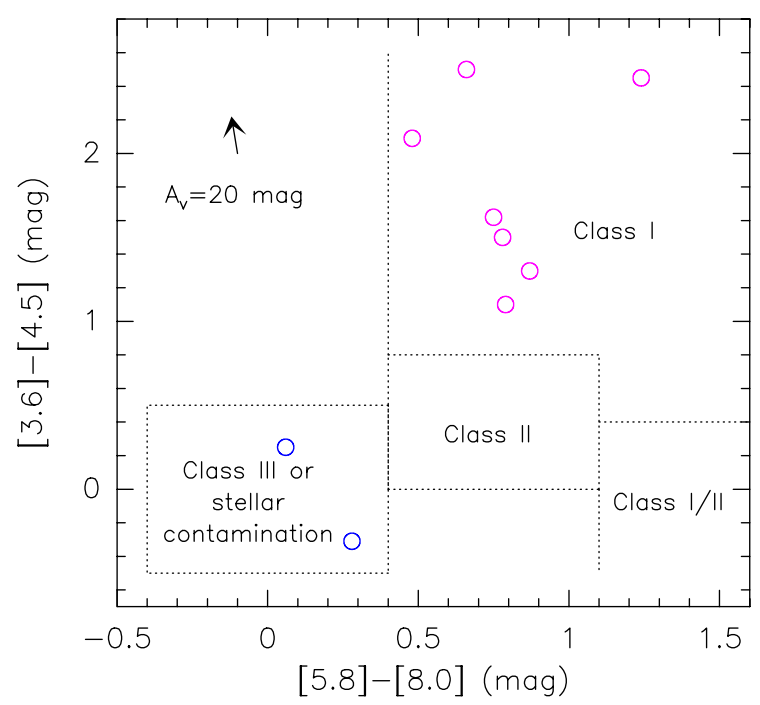

Fig. B.3. Mid-infrared [3.6]-[4.5] vs. [5.8]-[8.0] colour-colour diagram for the nine sources extracted at $8 \mu \mathrm{m}$. The colour coding is the same as in Fig. B.1. The arrow indicates an extinction of $A_{v}=10$. The different regions of the plot corresponding to the different protostellar classes are also shown. 